20 with osteoarthritis and 20 control participants. All patients completed the Fibromyalgia Impact Questionnaire. Sphygmomanometry was performed three times on both arms, and participants were asked to indicate whether the procedure was painful.

Allodynia was evoked in more patients in the fibromyalgia group than in the other groups; $69 \%$ versus $5 \%, 10 \%$ and $2 \%$ for participants in the rheumatoid arthritis, osteoarthritis and control groups, respectively. The mean blood pressure value at which allodynia occurred was also significantly lower in the fibromyalgia group than the other groups $(P<0.001)$. In patients with fibromyalgia, strong correlations were found between sphygmomanometry-evoked allodynia and the Fibromyalgia Impact Questionnaire score, and with the number of tender points.

This study had some limitations (e.g. one center lacked an independent assessor). Large, prospective studies are required to validate the use of blood sphygmomanometry in the diagnosis of fibromyalgia.

Original article Vargas A et al. (2006) Sphygmomanometryevoked allodynia - a simple bedside test indicative of fibromyalgia. J Clin Rheumatol 12: 272-274

\section{Increased SLE disease activity and duration do not worsen patients' cardiovascular risk}

Patients with systemic lupus erythematosus (SLE) have a strikingly high prevalence of atherosclerosis and risk of cardiovascular events, compared with the general population, but the 'lupus factor' responsible for this association remains to be identified. Maksimowicz-McKinnon and colleagues, therefore, looked for associations between SLE characteristics and markers of cardiovascular disease. Perhaps surprisingly, they found that SLE activity and duration were not associated with carotid atherosclerosis.

The authors looked for clinically significant atherosclerotic plaque in 605 participants (mean age 46.5 years; $91 \%$ female; $39 \%$ African American) in the Hopkins Lupus Cohort Study, who were prospectively followed at quarterly intervals. Age was strongly associated with carotid plaque; the prevalence of plaque was $1 \%$ in patients aged $<30$ years, and $61 \%$ in those aged $\geq 60$ years. After adjustment for age, carotid plaque was only associated with traditional cardiovascular risk factors: male sex, hypertension, diabetes mellitus, and high serum complement C3 and creatinine levels. The association of carotid plaque with C3 levels suggests that other measures of acute-phase inflammation (e.g. high-sensitivity C-reactive protein assay results) might reveal endothelial dysfunction or atherogenesis in patients with SLE.

The authors conclude that SLE patients at the greatest risk of atherosclerosis (those with hypertension or elevated serum C3 levels) are not those with the most active disease. Indeed, the authors suggest that patients with persistent, 'grumbling' SLE, who are often treated conservatively, could have an increased risk of coronary artery disease. They speculate that chronicity, rather than severity, of systemic inflammation might have the greatest effect on atherogenesis.

\section{Original article Maksimowicz-McKinnon K et al. (2006) Predictors of carotid atherosclerosis in systemic lupus erythematosus. J Rheumatol 33: 2458-2463 \\ Synovitis can be present even if Doppler ultrasound findings are negative}

Doppler ultrasound is thought to be more sensitive than gray-scale ultrasound in the detection of synovitis, because Doppler ultrasound can also detect perfusion and blood flow. The sensitivity of Doppler ultrasound is, however, devicedependent. Koski and colleagues found that only one of the three devices they evaluated (the Aplio 50 from Toshiba Medical Systems, Tokyo, Japan) was capable of detecting capillary flow $(<1 \mathrm{~mm} / \mathrm{s})$.

Koski and colleagues recruited 44 consecutive patients with monoarthritis or polyarthritis and clinical indications for synovial biopsy of a single site (e.g. small and large joints, bursae and tendon sheaths). Each site underwent an ultrasound scan and a percutaneous synovial biopsy. Histologic analysis showed active synovitis (i.e. leukocytes in the synovium) in 35 cases, 29 of which also showed a positive Doppler ultrasound signal. Other synovial histopathology was present in eight cases, five of which also showed a positive Doppler ultrasound signal. Negative findings on Doppler ultrasound did not, therefore, preclude the presence of synovitis, although a positive Doppler signal indicated active synovitis. 